\title{
Worry Loves Company, but Unnecessary Consultations May Harm the Patients We Comanage
}

\author{
James Anstey, MD*, Brian P Lucas, MD, MS²,34
}

\begin{abstract}
'Division of Hospital Medicine, Department of Medicine, University of California, San Francisco, California; ${ }^{2}$ Medicine Service, White River Junction VA Medical Center, White River Junction, Vermont; ${ }^{3}$ Geisel School of Medicine at Dartmouth College, Hanover, New Hampshire; ${ }^{4} T h e ~ D a r t m o u t h$ Institute for Health Policy \& Clinical Practice, Hanover, New Hampshire.
\end{abstract}

"Never worry alone" is a common mantra that most of us have heard throughout medical training. The premise is simple and well meaning. If a patient has an issue that concerns you, ask someone for help. As a student, this can be a resident; as a resident, this can be an attending. However, for hospitalists, the answer is often a subspecialty consultation. Asking for help never seems to be wrong, but what happens when our worry delays appropriate care with unnecessary consultations? In this month's issue of the Journal of Hospital Medicine, authors Bellas et al. have investigated this issue through the lens of subspecialty preoperative consultation for patients admitted to a hospitalist comanagement service with a fragility hip fracture requiring surgery. ${ }^{1}$

Morbidity and mortality for patients who experience hip fractures are high, and time to appropriate surgery is one of the few modifiable risk factors that may reduce morbidity and mortality. ${ }^{2,3}$ Bellas et al. conducted a retrospective cohort study to test the association between preoperative subspecialty consultation and multiple clinically relevant outcomes in patients admitted with an acute hip fracture. ${ }^{1}$ All patients were comanaged by a hospitalist and orthopedic surgery, and "consultation" was defined as any preoperative subspecialty consultation requested by the hospitalist. Outcome measures included time to surgery, length of stay, readmission rate, perioperative complications, and 30-day mortality. In total, 36\% (177/491) of patients who underwent surgery received a subspecialty preoperative consultation. Unsurprisingly, these patients were older with higher rates of comorbidity. After controlling for age and Charlson Comorbidity Index, preoperative consultation was associated with dramatic delays and increased rates of time to surgery $>24$ hours (adjusted odds ratio, 4.2; 95\% Cl: 2.8-6.6). The authors classified $90 \%$ of consultations as appropriate, either because of an active condition (eg, acute coronary syndrome) or because admitting physicians documented a perception that patients were at increased risk. However, $73 \%$ of consultations had only minor recommendations, such as ordering an ECG or changing the dose of an existing medication, and only $37 \%$ of the time did consultations lead to an identifiable change in management as a result of the consultation.

*Corresponding Author: James Anstey, MD; E-mail: james.anstey@ucsf.edu; Telephone: 209-606-1380; Twitter: @jeanstey

Received: July 29, 2019; Revised: August 9, 2019; Accepted: August 9, 2019

(c) 2020 Society of Hospital Medicine DOI 10.12788/jhm.3304
Although striking, integrating these findings into clinical practice is complex. As a retrospective study, patients who received consultations were obviously different from those who did not. The authors attempted to adjust for this but used only age and Charlson Comorbidity Index. Other factors that are both associated with consultations and known to increase mortality — such as frailty and functional status - were not included in their adjustment. Such unmeasured confounders possibly explain at least some, if not all, of the findings that consultations were associated with a doubling of the likelihood of 30day mortality. In addition, although the authors assessed the appropriateness of consultation and degree of recommendations, their methods for this deserve scrutiny. Two independent providers adjudicated the consultations with excellent agreement (kappa 0.96 for indication, 0.95 for degree of recommendation), but this reliability assessment was done on previously extracted chart data, probably inflating their agreement statistics. Finally, the adjudication of consultant recommendations into minor, moderate, and major categories may oversimplify the outcome of each consultation. For example, all medication recommendations, regardless of type, were considered as minor, and recommendations were considered as major only if they resulted in invasive testing or procedures. This approach may underrepresent the impact of consultations as in clinical practice not all high-impact recommendations result in invasive testing or procedures. Despite these important limitations, Bellas et al. present a compelling case for preoperative consultation being associated with delays in surgery.

How then should this study change practice? The authors' findings tell two separate but intertwined stories. The first is that preoperative consultation leads to delays in surgery. As patients who received preoperative consultation were obviously sicker, and because delays caused by consultation may lead to increased morbidity and mortality, perhaps the solution is to simply fix the delays. However, this approach ignores the more compelling story the authors tell. More important than the delays was the surprising lack of impact of preoperative consultations. Bellas et al. found that the majority of consultations resulted in only minor recommendations, and more importantly, hospitalists rarely changed treatment as a result. Although patients who received consultations were more ill, consultation rarely changed their care or decreased the risk posed by surgery. Bellas et al. found that only patients with active medical conditions had consultations, which resulted in moderate or major recommendations. These findings highlight an oppor- 
tunity to better identify patients for whom consultation might be helpful and to prevent delays by avoiding consultation for those unlikely to benefit. There have been several efforts in the orthopedic literature to use guidelines for preoperative cardiac testing to guide cardiology consultation. ${ }^{4,5,6}$ One study using this approach reported findings that were extremely similar to those reported by Bellas et al. in that $71 \%$ of preoperative cardiology consultations in their institution did not meet the guideline criteria for invasive cardiac testing. ${ }^{7}$ The primary difference between the findings of Bellas et al. and the studies in the orthopedic literature is the presence of the comanaging hospitalist. As more and more patients receive hospitalist comanagement prior to inpatient surgery, it is well within the scope of the hospitalist to differentiate chronic risk factors from active or decompensated medical disease requiring a subspecialist. This is in fact much of the value that a hospitalist adds. Avoiding consultation for patients with only elevated chronic risk factors is an important first step in avoiding unnecessary delays to surgery and an opportunity for hospitalists to improve the care of the patients they comanage.

The goal of teaching trainees to "never worry alone" is to harness the feelings of uncertainty that all providers face to improve patient care. Knowing when to worry is a valuable lesson, but as with all skills, it should be applied thoughtfully and informed by evidence. Appreciating the risks that surgery poses is quintessential to safe perioperative care, but equally important is understanding that inappropriate consultations can create risks from needless delays and testing. Only in bal- ancing these two concerns, and appreciating when it is appropriate to worry, can we provide the highest quality of care to our patients.

Disclosures: The authors declare that they have nothing to disclose.

Funding: Department of Veterans Affairs, Veterans Health Administration,

Office of Research and Development and Dartmouth SYNERGY, National Institutes of Health, National Center for Translational Science (UL1TR001086).

\section{References}

1. Bellas N, Stohler S, Staff I, et al. Role of preoperative consults and hospitalist comanagement in hip fracture patients. J Hosp Med. 2020;15(1):16-21. https:doi.org/jhm.3264.

2. Goldacre MJ, Roberts SE, Yeates D. Mortality after admission to hospital with fractured neck of femur: database study. BMJ 2002;325(7369):868-869. https://doi.org/10.1136/bmj.325.7369.868.

3. Shiga T, Wajima Z, Ohe Y. Is operative delay associated with increased mortality of hip fracture patients? Systematic review, meta-analysis, and meta-regression. Can J Anaesth. 2008;55(3):146-154. https://doi.org/10.1007/ BF03016088.

4. Cluett J, Caplan J, Yu W. Preoperative cardiac evaluation of patients with acute hip fracture. Am J Orthop. 2008;37(1):32-36.

5. Smeets SJ, Poeze M, Verbruggen JP. Preoperative cardiac evaluation of geriatric patients with hip fracture. Injury. 2012;43(12):2146-2151. https://doi. org/10.1016/j.injury.2012.08.007.

6. Siu CW, Sun NC, Lau TW, Yiu KH, Leung F, Tse HF. Preoperative cardiac risk assessment in geriatric patients with hip fractures: an orthopedic surgeons' perspective. Osteoporos Int. 2010;21(Suppl 4):S587-S591. https://doi. org/10.1007/s00198-010-1393-0.

7. Stitgen A, Poludnianyk K, Dulaney-Cripe E, Markert R, Prayson M. Adherence to preoperative cardiac clearance guidelines in hip fracture patients. J Orthop Trauma 2015;29(11):500-503. https://doi.org/10.1097/ BOT.0000000000000381 